\title{
Syndrome de manque induit par l'interaction médicamenteuse entre nalméfène et méthadone
}

\author{
Drug-Induced Withdrawal Syndrome after Co-medication of Nalmefene and Methadone
}

\author{
S. Cucchi $\cdot$ L. Gallucci $\cdot$ J. Reynoard \\ Reçu le 12 février 2018; accepté le 14 mai 2018 \\ (C) SFMU et Lavoisier SAS 2018
}

\section{Introduction}

Le nalméfène est un inhibiteur des opioïdes autorisé en Europe depuis 2013 dans le traitement de la dépendance alcoolique $[1,2]$. C'est un antagoniste sélectif des récepteurs $\mu$ et $\delta$-opioïdes et un agoniste partiel des récepteurs $\kappa$ opioïdes comme la naltrexone, mais possédant une demivie plasmatique plus longue [2]. Cette molécule a été utilisée en médecine d'urgence pendant plusieurs années comme antidote en cas d'overdose aux opiacés. Sa structure chimique est très proche de celle de la naloxone qui l'a supplantée dans cette indication par sa facilité d'utilisation et sa durée d'action plus courte [1,3]. La méthadone est un agoniste opioïde fréquemment utilisé pour le traitement substitutif de la dépendance aux opiacés [4]. Elle est rapidement absorbée, et le pic plasmatique est atteint entre deux et quatre heures avec une durée d'action longue [7]. La capacité d'un antagoniste à induire un syndrome de manque est fondée sur l'inhibition compétitive des récepteurs opioïdes, dépend de la nature des substances, de la dose quotidienne utilisée et de la dose d'antagoniste [5]. Ce cas clinique rapporte un tableau de syndrome de manque sévère induit par la prise concomitante de nalméfène et de méthadone.

\section{S. Cucchi $(\bowtie)$}

Service d'accueil des urgences, polyclinique du Sud de la Corse, F-20137 Porto-Vecchio, France

e-mail : sabrina.cucchi@hotmail.fr

L. Gallucci

Samu 2A, centre hospitalier de la Miséricorde,

27, avenue Impératrice-Eugénie,

F-20000 Ajaccio, France

J. Reynoard

Centre antipoison et de toxicovigilance de Marseille, hôpital Sainte-Marguerite, 270, boulevard de Sainte-Marguerite, F-13274 Marseille cedex 09, France

\section{Observation}

Un homme de 26 ans est admis aux urgences par le Smur pour un état d'agitation, des douleurs abdominales, des diarrhées et des vomissements ; il est décrit comme toxicomane connu sous traitement substitutif par méthadone $(80 \mathrm{mg} / \mathrm{j})$ et oxazépam $50 \mathrm{mg}$ depuis plusieurs mois. L'appel initial au Samu a été passé par sa petite amie pour des douleurs abdominales et vomissements survenus brutalement depuis quelques heures avec agitation. La prise en charge Smur a consisté en la pose d'une voie veineuse périphérique, une hydratation par chlorure de sodium, un traitement symptomatique intraveineux par paracétamol $1 \mathrm{~g}$, phloroglucinol $80 \mathrm{mg}$, ésoméprazole $40 \mathrm{mg}$ et métoclopramide $10 \mathrm{mg}$ ainsi qu'une sédation par clorazépate $20 \mathrm{mg}$, hydroxizine $100 \mathrm{mg}$ et midazolam $3 \mathrm{mg}$. Une ordonnance de nalméfène $18 \mathrm{mg}$ datée de cinq jours plus tôt est également retrouvée au domicile.

À son arrivée, le patient était conscient, un score de Glasgow à 15 mais totalement obnubilé, confus, désorienté, présentant une agitation psychomotrice, des hallucinations et des propos délirants ainsi que des clonies diffuses et des sueurs profuses. Les constantes montraient une hyperthermie à $38,5^{\circ} \mathrm{C}$, une polypnée à 30 cycles/minute, une fréquence cardiaque à 130 battements/minute et une tension artérielle à 125/85 mmHg. La glycémie capillaire était à $1,20 \mathrm{~g} / 1$ et la saturation en oxygène à $100 \%$ sous lunettes à $3 \mathrm{l} / \mathrm{min}$. L'examen somatique ne montrait pas de signe de traumatisme crânien ni de focalisation neurologique ; l'abdomen était difficilement examinable du fait d'une hypertonie globale du patient, mais sans défense ni contracture abdominale. Le patient présentait des bâillements répétés et de nombreuses débâcles diarrhéiques non glairosanglantes.

Devant l'agitation importante, limitant la prise en charge et induisant un risque traumatique, une contention physique a été nécessaire ; la titration intraveineuse de midazolam a été poursuivie (10 mg en tout) ainsi que diazépam $20 \mathrm{mg}$ permettant une sédation progressive. Le traitement antalgique a été prolongé par néfopam $20 \mathrm{mg}$. 
La gazométrie artérielle ne montrait pas de troubles métaboliques ; le bilan biologique était normal, sans syndrome inflammatoire ; l'alcoolémie était négative. Le dosage des toxiques sanguins n'était pas réalisable au laboratoire local et la recherche urinaire ne rendant que des résultats qualitatifs sur morphiniques, benzodiazépines, cocaïne et cannabis, elle n'a pas été jugée pertinente du fait des traitements reçus par le patient et de son traitement habituel.

Après un interrogatoire insistant de l'entourage du patient, nous avons retrouvé la notion de douleurs épigastriques récentes pour lesquelles il avait consulté un médecin de ville cinq jours avant ; pour l'aider à diminuer sa consommation d'alcool à l'origine de ces douleurs, un traitement par nalméfène lui avait été prescrit. Il l'avait débuté le matin même, sa petite amie décrivant un début des signes dans les minutes qui ont suivi la prise d'un comprimé à $18 \mathrm{mg}$.

Un avis auprès du centre antipoison de Marseille a confirmé la contre-indication d'utilisation concomitante de méthadone et de nalméfène, du fait de l'interaction spécifique de ces molécules sur les récepteurs opioïdes, induisant ce tableau de syndrome de manque sévère. La prise en charge thérapeutique consistant à traiter le patient avec de fortes doses d'opiacés sur plusieurs jours du fait de la demi-vie et de la durée d'action longues du nalméfène, il a été décidé d'un transfert vers le centre hospitalier d'Ajaccio, disposant d'un service de soins intensifs et de réanimation adapté. Après un transfert médicalisé par le Smur, le patient a séjourné 48 heures en réanimation où un traitement par midazolam intraveineux a permis la régression du tableau sans complications, puis il a été pris en charge en service d'addictologie. Une déclaration de pharmacovigilance a été faite par le centre antipoison de Marseille.

\section{Discussion}

La prise en charge du syndrome de manque aux urgences est complexe tant au niveau diagnostique que thérapeutique, car il s'agit de patients souvent instables dont l'histoire de la maladie et les prises médicamenteuses exactes sont difficiles à établir à la phase aiguë. Les médecins urgentistes doivent être très attentifs dans le repérage des signes évocateurs de syndrome de manque ainsi que dans la notion de prise médicamenteuse avec ou sans supervision médicale [6]. Les principaux symptômes du syndrome de sevrage sont des yeux vitreux, une rhinorrhée, des bâillements, des sueurs profuses, une agitation, une irritabilité ou agressivité, des trémulations, des nausées, des vomissements, des diarrhées, une hypertension artérielle, des crampes et douleurs musculaires pouvant durer jusqu'à sept jours selon la nature du produit utilisé [1].

L'interaction entre nalméfène et méthadone s'explique par leur action compétitive sur les récepteurs opioïdes. L'ef- fet de la méthadone, agoniste des récepteurs opioïdes- $\mu$, est aboli par la fixation du nalméfène sur ces récepteurs avec une meilleure affinité et une durée de fixation plus longue [7]. Le délai de survenue des symptômes après l'ingestion de nalméfène varie de quelques minutes à une heure du fait d'une absorption rapide, le pic plasmatique étant atteint dans les 90 minutes [1]. De plus, du fait d'une demi-vie d'élimination longue (13,4 heures après une seule dose), le taux d'occupation des récepteurs opioïdes 50 heures après une ingestion unique ou répétée est de 48,4 à $72 \%$ [1,7], expliquant le caractère prolongé du syndrome de manque.

Dans notre cas, les hallucinations et le syndrome délirant ne rentrent pas dans le tableau classique du syndrome de sevrage ; ils sont expliqués par l'action agoniste du nalméfène sur les récepteurs $\kappa$, rendant la présentation clinique plus difficilement identifiable. Cette action, qui permet de réduire l'effet « récompense » addictive de la consommation d'alcool, peut donner des hallucinations (auditives, tactiles, visuelles et somatiques) ou dissociation, rapportées dans le résumé des caractéristiques du produit à une fréquence indéterminée et décrites dans une revue de la littérature sur les propriétés pharmacologiques du nalméfène [8] comme modérées et de courte durée, en lien avec l'initiation du traitement.

Plusieurs cas de syndromes de manque induits par la prise de nalméfène chez des patients dépendants aux opioïdes ont été décrits dans la littérature [1] ; une équipe française a récemment publié une série de 11 cas en Lorraine [9]. Une étude allemande [10] rapporte que sur les 200 réactions indésirables du nalméfène documentées dans le monde (base de données de pharmacovigilance de l'OMS entre le 7 mars 1997 et le $1^{\mathrm{er}}$ mars 2015), le nalméfène a été administré avec un opioïde dans 21 cas $(10,5 \%)$, provoquant des symptômes de sevrage.

La prise en charge de ce syndrome de manque consiste en une délivrance intraveineuse d'agonistes $\mu$-opioïdes puissants et de longue durée d'action [1]. Chez les patients consommateurs de méthadone ou d'autre agonistes $\mu$ opioïdes, certains auteurs préconisent l'utilisation d'une dose de charge de 10-20 mg de méthadone qui pourrait supprimer les symptômes, puis en fonction de l'évolution une seconde dose quatre à six heures plus tard [1]. Dans la série rapportée par Franchitto et al. [1], les molécules utilisées pour supprimer le syndrome de manque étaient de la méthadone, des benzodiazépines, du midazolam, de la morphine, de la clonidine et de la thiamine.

Il est important d'orienter secondairement le patient vers une structure de soins spécialisés, car l'utilisation de nombreuses substances au cours du traitement peut entraîner un état de dépendance pouvant conduire à des automédications ou un mésusage de médicaments. Les personnes développant une dépendance aux opiacés ont fréquemment des troubles de consommation alcoolique associés. La prescription 
de nalméfène doit être prudemment évaluée, et les praticiens doivent s'assurer de l'absence de consommation d'opioïdes (dépendance, traitement substitutif ou mésusage) chez ces patients avant de prescrire cette molécule [1].

En conclusion, le syndrome de manque induit par l'interaction entre nalméfène et méthadone est sévère et prolongé du fait de la demi-vie longue du nalméfène. La démarche diagnostique et thérapeutique chez ces patients est complexe aux urgences, et le traitement repose sur l'administration de puissants opiacés en milieu sécurisé et nécessite un relais de prise en charge avec des structures adaptées. Ce risque évitable impose une plus grande vigilance des médecins prescripteurs sur les associations médicamenteuses, les comorbidités ou les dépendances associées.

Conflits d'intérêts : les auteurs déclarent ne pas avoir de liens d'intérêts.

\section{Références}

1. Franchitto N, Jullian B, Salles J, et al (2017) Management of precipitated opiate withdrawal syndrome induced by nalmefene mistakenly prescribed in opiate-dependent patients: a review for clinicians. Expert Opin Drug Metab Toxicol 13:669-77
2. Mann K, Torup L, Sørensen P, et al (2016) Nalmefene for the management of alcohol dependence: review on its pharmacology, mechanism of action and meta-analysis on its clinical efficacy. Eur Neuropsychopharmacol 26:1941-49

3. Kaplan JL, Marx JA, Calabro JJ, et al (1999) Double-blind randomized study of nalmefene and naloxone in emergency department patients with suspected narcotic overdose. Ann Emerg Med 34:42-50

4. Bart G (2012) Maintenance medication for opiate addiction: the foundation of recovery. J Addict Dis 31:207-25

5. Leri F (2008) Co-administration of opioid agonists and antagonists in addiction and pain medicine. Expert Opin Pharmacother 9:1387-96

6. Haber PS, Demirkol A, Lange K, et al (2009) Management of injecting drug users admitted to hospital. Lancet 374:1284-93

7. Ingman K, Hagelberg N, Aalto S, et al (2005) Prolonged central $\mu$-opioid receptor occupancy after single and repeated nalmefene dosing. Neuropsychopharmacology 30:2245-53

8. Paille F, Martini H (2014) Nalmefene: a new approach to the treatment of alcohol dependence. Subst Abuse Rehabil 5:87-94

9. Yéléhé-Okouma M, Martini H, Lemarié J, et al (2017) Opioid substitution therapy or hidden opioids are a minefield for nalmefene: an atypical case series of 11 patients in Lorraine. Fundam Clin Pharmacol 31:574-79

10. Dahmke H, Kupferschmidt H, Kullak-Ublick GA, et al (2015) Nalmefene and opioid withdrawal syndrome: analysis of the global pharmacovigilance database for adverse drug reactions. Praxis 104:1129-34 